\title{
Antifungal resistant isolates of Candida glabrata from the United States are enriched for specific sequence types with distinct $\mathrm{MSH} 2$ alleles
}

RUTGERS

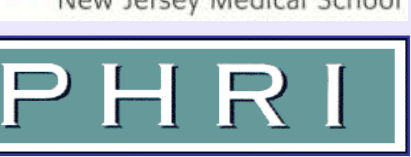

\author{
K.R. Healey*, E. Shor, and D. S. Perlin
}

Public Health Research Institute, New Jersey Medical School, Rutgers University, Newark, NJ, USA ${ }^{*}$ Corresponding author: Kelley R. Healey, Ph.D. +1 973-854-3202 email: krh75@njms.rutgers.edu

Rates of acquired resistance to triazoles and echinocandins are substantially higher among strains of Candida glabrata compared to other Candida species, particularly in the U.S. We previously demonstrated that disruption of the DNA mismatch repair (MMR) gene $M S H 2$ increases $C$. glabrata antifungal resistance frequencies. Here, we feature the diversity of $M S H 2$ polymorphisms identified in strains from around the globe and highlight their associated sequence types and relevance in resistance potential. We have performed multilocus sequence typing (MLST) on 110 U.S. strains and have found that echinocandin (ECH)-, fluconazole (FLC)-, and multidrug (MDR)- resistant strains are enriched for sequence types (STs) 10, 16, 8, 3, and 6, while susceptible isolates are comprised of a more diverse ST array. ST10, ST16, and ST8 are associated with partial loss of function Msh2 alleles that carry amino acid substitutions P208S/N890I, E231G/L269F, and V239L/A942T, respectively. Interestingly, ST10 and ST16 (and therefore their associated Msh2 alleles) are almost exclusively found within U.S. isolates. Other Msh2 alleles, primarily identified in international isolates, imparted partial (e.g. S346T, M651T) or no (e.g. E456D, E459K, R847C) Msh2 LOF. Greater genetic diversity in terms of MSH2 sequences and STs (including many novel STs) were identified within international isolates.
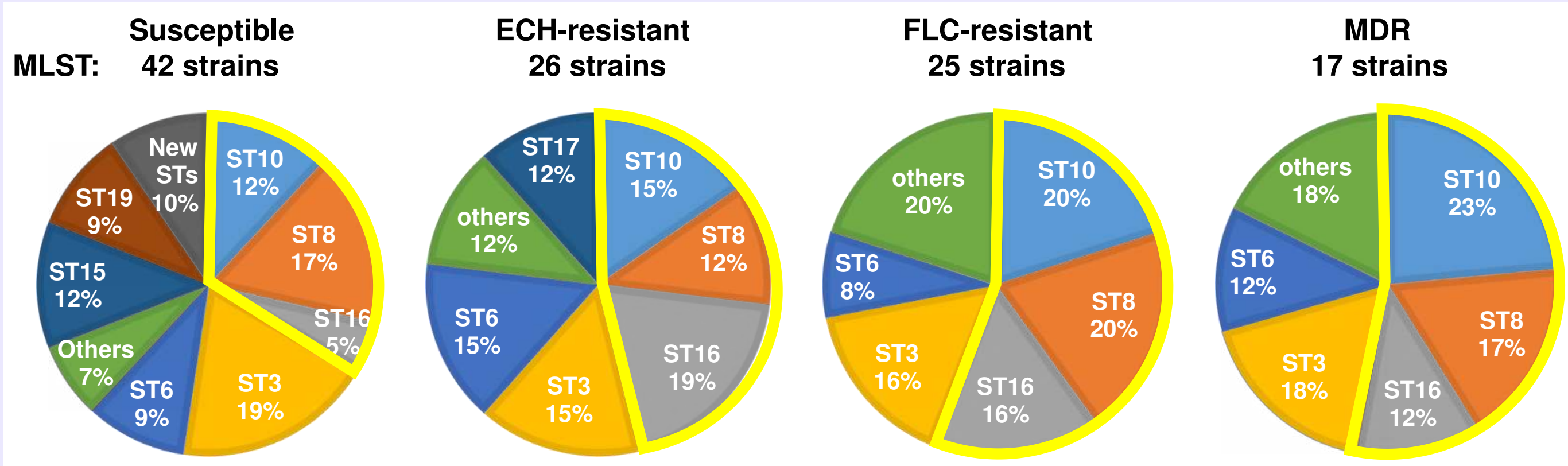

Figure 1. Sequence type (ST) distribution of U.S.-based isolates. - Resistant isolates from the U.S.
are comprised of fewer STs than the susceptible isolate population (Fig. 1).

- STs 8, 10, and 16 contain partial LOF Msh2 alleles (yellow highlight).

- STs 10 and 16 are nearly exclusive to U.S. isolates.

- Non-U.S. isolates demonstrate greater ST (incl. new STs) and Msh2 profile diversity (Table 1).

Table 1. List of identified MSH2 genotypes and associated geographical locations, phenotype, and sequence type(s)

\begin{tabular}{|c|c|c|c|}
\hline MSH2 genotype & Location(s) & LOF phenotype* & Sequence Type(s) \\
\hline WT (as ATCC 2001) & $1,2,3,4,5,7,8$ & - & $3,6,15,17,19,59$ \\
\hline E7K & 6,8 & - & 3 \\
\hline P27L/I156L & 8 & unknown & new \\
\hline P208S/N890I & $1,2,3,6,8$ & +++ & 10 \\
\hline P208S/N890I/Y949C & 6 & +++ & . \\
\hline L269F & $1,2,3$ & ++ & \\
\hline E231G/L269F & $1,2,3,4$ & ++ & 16 \\
\hline E231G/L269S & 3 & unknown & 16 \\
\hline V239L & $1,2,3,4,5,7,8$ & ++ & 46,7, new \\
\hline V239L/A942T & $1,2,3,4,5,6,8$ & ++ & 8,2 \\
\hline V239L/S757T & 2 & unknown &. \\
\hline V239L/D946E & 7 & unknown & . \\
\hline V34E/V239L/A942T & 6 & unknown & . \\
\hline V239L/K814E/A942T & 5 & unknown & . \\
\hline $\mathrm{Q} 259 \mathrm{H}$ & 4 & unknown & new \\
\hline S346T & 6 & + & . \\
\hline Q386K & 7 & +++ & \\
\hline E456D & $1,2,3,6,8$ & - & new \\
\hline E459K & 7,8 & - & 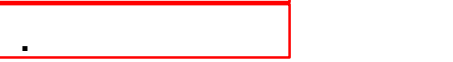 \\
\hline $\mathrm{E} 478 \mathrm{Q}$ & 2,6 & ++ & . \\
\hline S591Y & 6 & +++ & . \\
\hline E608D & 8 & unknown & . \\
\hline M651T & 6 & + & . \\
\hline T772S & 7 & unknown & . \\
\hline $\mathrm{R} 847 \mathrm{C}$ & 7 & - & . \\
\hline V856A & 8 & unknown & new \\
\hline
\end{tabular}

Center locations: 1: U.S. (TX); 2: U.S. (GA/MD/TN); 3: U.S. (NC); 4: U.S. (MI); 5: Europe 1;

6: Europe 2; 7: South Asia; 8: Middle East

*Phenotype definition

(see definitions at right and Fig. 2 top)

MSH2 genotype identified in at least 10 isolates
$=1-2$ fold increase

$+=3-4$ fold increase

$++=5-8$ fold increase

$+++=\geq 9$ fold increase

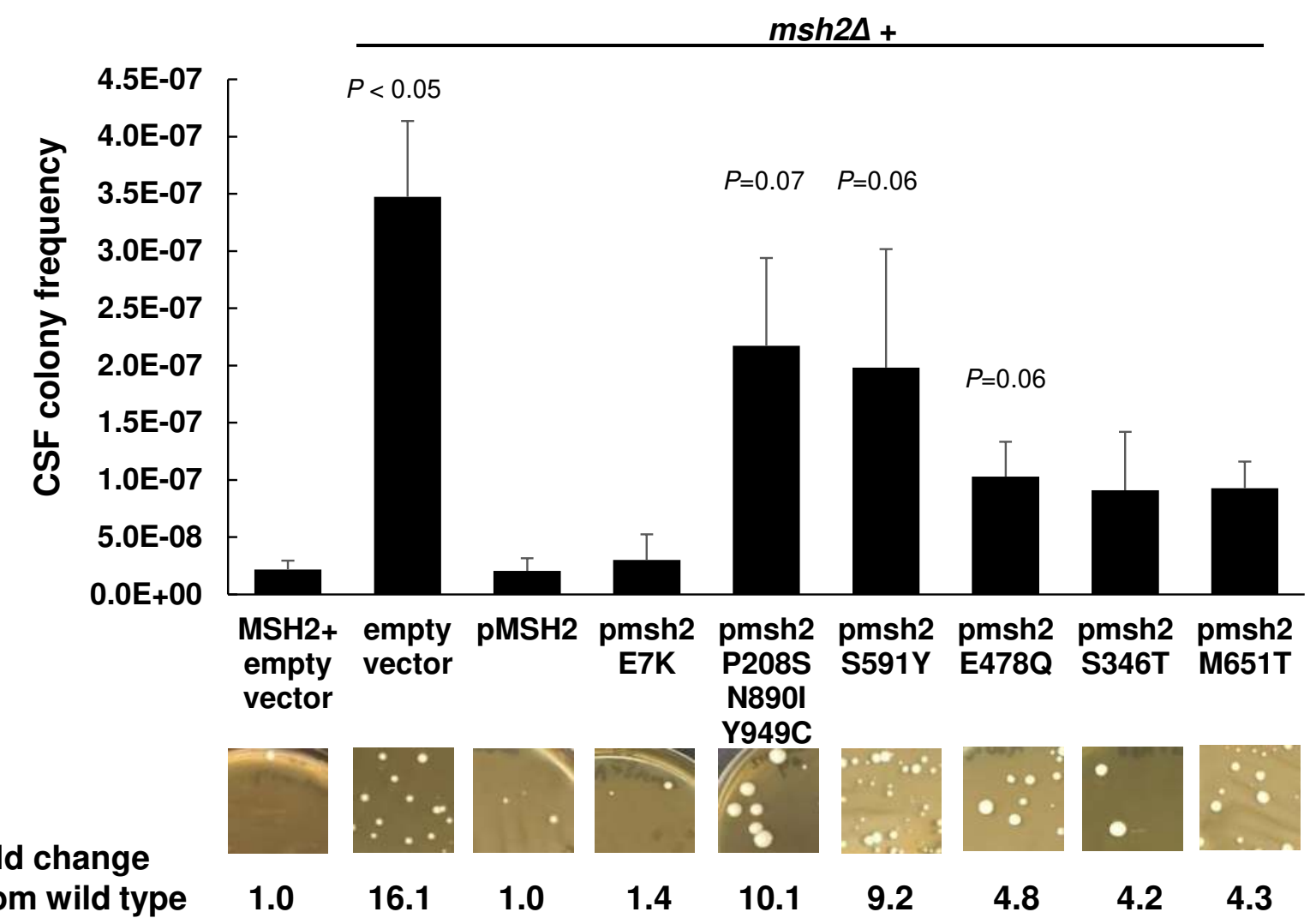

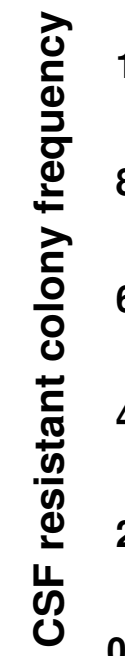

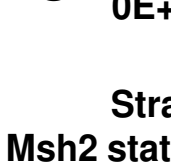

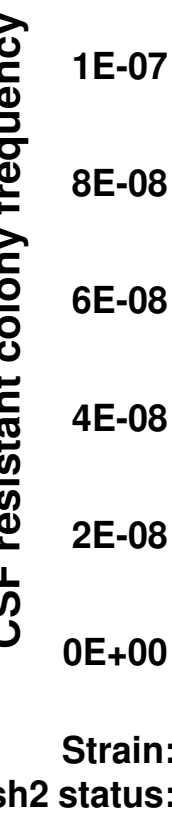

10.1

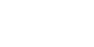

